PROCEEDINGS OF THE

AMERICAN MATHEMATICAL SOCIETY

Volume 129, Number 5, Pages 1445-1451

S 0002-9939(00)05950-5

Article electronically published on December 13, 2000

\title{
STURMIAN SEQUENCES AND THE LEXICOGRAPHIC WORLD
}

\author{
SHAOBO GAN
}

(Communicated by Michael Handel)

\begin{abstract}
In this paper, we give a complete description for the lexicographic world $\mathcal{L}=\left\{(x, y) \in \Sigma \times \Sigma: \Sigma_{x y} \neq \emptyset\right\}=\{(x, y): y \geq \phi(x)\}$, where $\Sigma=$ $\{0,1\}^{\mathbf{N}}, \Sigma_{a b}=\left\{x \in \Sigma: a \leq \sigma^{i}(x) \leq b\right.$, for all $\left.i \geq 0\right\}, \phi: \Sigma \rightarrow \Sigma$ is defined by $\phi(a)=\inf \left\{b: \Sigma_{a b} \neq \emptyset\right\}$ and the order $\leq$ is the lexicographic order on $\Sigma$. The main result is that $b=\phi(a)$ for some $a=0 x$ if and only if $b$ is the Sturmian sequence $b$ such that $\operatorname{Orb}(b) \subset[0 x, 1 x]$ and $\sigma^{i}(b) \leq b$ for all $i \geq 0$. At the same time, a new description of Sturmian minimal sets is given. A minimal set $M$ is a Sturmian minimal set if and only if, for some $x \in \Sigma, M \subset[0 x, 1 x]$. Moreover, for any $x \in \Sigma$, there exists a unique Sturmian minimal set in $[0 x, 1 x]$.
\end{abstract}

\section{INTRODUCTION AND THE DEFINITION OF LEXICOGRAPHIC WORLD}

Let $\Sigma=\{0,1\}^{\mathbf{N}}$ and denote by $\sigma$ the left (one-sided) shift on $\Sigma$.

First, let us define the lexicographic order on $\Sigma$. For any $x, y \in \Sigma, x<y$ iff $x \neq y$ and for some $n \in \mathbf{N}, x_{i}=y_{i}$ for $i<n$ and $x_{n}=0, y_{n}=1$. Note that $\Sigma$ is well-ordered and the order topology given by the above order is the same as the usual topology on $\Sigma$.

For any $a, b \in \Sigma$ define $\Sigma_{a b}$ as

$$
\begin{aligned}
\Sigma_{a b} & =\left\{x \in \Sigma: a \leq \sigma^{i}(x) \leq b, \text { for all } i \geq 0\right\} \\
\mathcal{L} & =\left\{(x, y) \in \Sigma \times \Sigma: \Sigma_{x y} \neq \emptyset\right\} .
\end{aligned}
$$

For any $a, b \in \Sigma$ denote $\{x: a \leq x \leq b\}$ by $[a, b]$, which will be called a (closed) interval. And for any interval $I=[a, b]$, denote $\Sigma_{a b}$ by $\Sigma_{I}$. If $\Sigma_{I} \neq \emptyset$, I will be called an $\mathcal{L}$-interval. Now we define a map $\phi: \Sigma \rightarrow \Sigma$ as

$$
\phi(a)=\inf \left\{b \in \Sigma: \Sigma_{a, b} \neq \emptyset\right\} .
$$

Then $\mathcal{L}=\{(x, y): y \geq \phi(x)\}$ (Lemma 2.1). $\mathcal{L}$ is called the lexicographic world and it is closely related to the bifurcation of a Lorenz-like map (see [2]). In a talk, Labarca raised the question of studying $\phi$. In this paper, we give a complete description for $\phi$. The main result is

Theorem 1.1. $b=\phi(a)$ for some $a=0 x$ if and only if $b$ is the Sturmian sequence $b$ such that $\operatorname{Orb}(b) \subset[0 x, 1 x]$ and $\sigma^{i}(b) \leq b$ for all $i \geq 0$.

At the same time, we obtain a new description for Sturmian minimal sets.

Received by the editors August 21, 1999.

2000 Mathematics Subject Classification. Primary 37B10.

Key words and phrases. Sturmian sequences, lexicographic world.

This research was supported by the NSFC (No. 10001003) and Scientific Foundation for Returned Overseas Chinese Scholars, Ministry of Education.

(C)2000 American Mathematical Society 
Theorem 1.2. A minimal set $M$ is a Sturmian minimal set if and only if, for some $x \in \Sigma, M \subset[0 x, 1 x]$. Moreover, for any $x \in \Sigma$, there exists a unique Sturmian minimal set in $[0 x, 1 x]$.

This paper is organized as following. In $\S 2$, some basic properties of $\phi$ are given. And then we introduce the concept of order minimality in $\S 3$. It is shown that $b=\phi(a)$ if and only if $b$ is order minimal and $\sigma^{i}(b) \leq b$ for $i \geq 0$. In $\S 4$, we show that $b=\phi(a)$ is a Sturmian sequence. The main result and the characterization of Sturmian minimal sets are given in $\S 5$.

\section{Some basic properties of $\phi$}

Lemma 2.1. $\Sigma_{a \phi(a)} \neq \emptyset$. Therefore, $\mathcal{L}=\{(x, y) \in \Sigma \times \Sigma: y \geq \phi(x)\}$.

Proof. Let $b^{i} \in\left\{b \in \Sigma: \Sigma_{a, b} \neq \emptyset\right\}$ and $b^{i} \searrow \phi(a)$. This means $\exists x^{i} \in \Sigma_{a b^{i}}$, i.e.,

$$
a \leq \sigma^{n}\left(x^{i}\right) \leq b^{i}, \quad \forall i, n
$$

We may assume $x^{i} \rightarrow x$. Letting $i$ tend to $\infty$ we get

$$
a \leq \sigma^{n}(x) \leq \phi(a) \text {. }
$$

This means that $x \in \Sigma_{a \phi(a)}$.

Lemma 2.2. $\phi$ is non-decreasing.

Proof. If $a \leq b \in \Sigma$, then $\Sigma_{b \phi(b)} \neq \emptyset$ implies $\Sigma_{a \phi(b)} \neq \emptyset$. So $\phi(a) \leq \phi(b)$.

Lemma 2.3. $\phi$ is continuous from the left, i.e., if $a^{i}$ tends to a monotonically increasingly, then $\phi\left(a^{i}\right)$ tends to $\phi(a)$.

Proof. Let $\phi\left(a^{i}\right) \nearrow y$ and $x^{i} \in \Sigma_{a^{i} \phi\left(a^{i}\right)}, x^{i} \rightarrow x$. Then

$$
a^{i} \leq \sigma^{n}\left(x^{i}\right) \leq \phi\left(a^{i}\right), \quad \forall i, n .
$$

Letting $i \rightarrow \infty$,

$$
a \leq \sigma^{n}(x) \leq y
$$

So $\Sigma_{a y} \neq \emptyset$. This implies $\phi(a) \leq y$. But obviously $\phi(a) \geq y$ since $a \geq a^{i}$ and $\phi(a) \geq \phi\left(a^{i}\right)$. This proves $\phi(a)=y$.

Lemma 2.4. 1. $\phi\left(0^{\infty}\right)=0^{\infty}$.

2. $\phi\left(01^{\infty}\right)=1^{\infty}$.

3. $\phi(1 x)=1^{\infty}$, for any $x \in \Sigma$.

Proof. 1. Since $0^{\infty} \in \Sigma_{0^{\infty}, 0^{\infty}}, \phi\left(0^{\infty}\right)=0^{\infty}$.

2. Assume $01^{\infty} \leq \sigma^{n}(x) \leq 1^{\infty}, \forall n$ and $x=0^{n_{1}} 1^{l_{1}} 0^{n_{2}} 1^{l_{2}} \ldots$. If $n_{1}>0$, then $n_{1}=1$ and $n_{i}=0$ for $i>1$. If $n_{1}=0\left(l_{1}>0\right)$, then either $n_{i}=0$ for all $i$ or $n_{1}=n_{2}=\cdots=n_{k}=0$ and $n_{k+1}>0$, which implies $n_{k+i}>0$ for all $i>1$.

So for some $m$ such that $\sigma^{m}(x)=1^{\infty}$. This means $\phi\left(01^{\infty}\right)=1^{\infty}$.

3 . This is a corollary of 2 . and Lemma 2.2 .

Lemma 2.5. Let $a=w 01^{\infty}$ and $a^{\prime}=w 10^{\infty}$. Then $\phi(a)=\phi\left(a^{\prime}\right)$. Moreover if $b=w 10^{m} 1 v$ and $m>|w|$, then $\phi(b)=\phi(a)$. 
Proof. We only prove the second conclusion. The first is just a consequence of the second.

Take $x \in \Sigma_{a \phi(a)}$. If $\sigma^{l}(x) \geq b$ for all $l \geq 0$, then $x \in \Sigma_{b \phi(a)}$. Hence $\phi(b)=\phi(a)$. We will show that if there is $l \geq 0$ such that $a \leq \sigma^{l}(x)<b$, then with $k=|w|$ and $n=l+2(k+1) \sigma^{n}(x)=1^{\infty}$. This implies $\phi(a)=1^{\infty}$ and so $\phi(a)=\phi(b)$.

If $\sigma^{l}(x)=a$, then $\sigma^{n}(x)=1^{\infty}$. On the other hand if $\sigma^{l}(x)>a$, then $\sigma^{l}(x)=$ $w 10^{m} u \sigma^{l+k+1}(x)=0^{m} u \geq a=w 01^{\infty}$. Since $m>k$, we have $m=k+1$ and $u=1^{\infty}$ and hence $\sigma^{n}(x)=u=1^{\infty}$.

Corollary 2.6. $\phi$ is continuous at $a=w 01^{\infty}$ and $a^{\prime}=w 10^{\infty}$.

\section{Ordering minimality and the image of $\phi$}

For any $x \in \Sigma$ denote $I(x)=[i(x), s(x)]=\{y \in \Sigma: i(x) \leq y \leq s(x)\}$, where $i(x)=\inf \overline{\operatorname{Orb}(x)}$ and $s(x)=\sup \overline{\operatorname{Orb}(x)}$ and the infimum and supremum are taken with respect to the order $\leq$. Obviously, $I(x)$ is an $\mathcal{L}$-interval. A point $x \in \Sigma$ is called order minimal if $I(x) \supset I(y)$ then $I(x)=I(y)$. An $\mathcal{L}$-interval is called minimal if it has no proper $\mathcal{L}$-subinterval. Note that if $I$ is a minimal $\mathcal{L}$-interval, then every point in $\Sigma_{I}$ is order minimal.

Lemma 3.1. Every $\mathcal{L}$-interval contains a minimal $\mathcal{L}$-interval. Therefore, for any $x \in \Sigma$ there is an order minimal $y \in \Sigma$ such that $I(y) \subset I(x)$.

Proof. The inclusion relation on subsets gives a natural partial order on the set of all $\mathcal{L}$-intervals. For any totally ordering $\mathcal{L}$-intervals $\left\{I_{\alpha}\right\}$ let $I=\bigcap_{\alpha} I_{\alpha}$.

$$
\begin{aligned}
\Sigma_{I} & =\bigcap_{l} \sigma^{-l} I=\bigcap_{l} \sigma^{-l} \bigcap_{\alpha} I_{\alpha} \\
& =\bigcap_{\alpha} \bigcap_{l} \sigma^{-l} I_{\alpha}=\bigcap_{\alpha} \Sigma_{I_{\alpha}} \neq \emptyset .
\end{aligned}
$$

So $I$ is also an $\mathcal{L}$-interval.

According to Zorn's Lemma, the first conclusion of the lemma is correct.

Since $I(x)$ is an $\mathcal{L}$-interval, $I(x)$ contains a minimal $\mathcal{L}$-interval $I$. Then every point $y \in \Sigma_{I}$ is order minimal such that $I(y)=I \subset I(x)$.

Lemma 3.2. If $b=\phi(a)$, then $\sigma^{j}(b) \leq b, \forall j$.

Proof. Since $b=\phi(a)$, there exists $x \in \Sigma_{a b}$. Let $I(x)=[c, d]$. Then obviously, $c, d \in \Sigma_{a b}$. If $d<b$, then $\phi(a) \leq d<b$. So $b=d \in \Sigma_{a b}$.

Lemma 3.3. If $b=\phi(a)$, then $\overline{\operatorname{Orb}(b)}$ is a minimal set.

Proof. For any $x \in \overline{\operatorname{Orb}(b)}$, write $I(x)=[c, d]$. According to the definition of $\phi$, $b=d$. This means that $b \in \overline{\operatorname{Orb}(x)}$. And hence $\overline{\operatorname{Orb}(b)} \subset \overline{\operatorname{Orb}(x)}$. This proves the result.

Theorem 3.4. $b=\phi(a)$ if and only if $\sigma^{j}(b) \leq b, j \geq 0$ and $b$ is order minimal.

Proof. " $\Rightarrow$ ". We only need to show $b$ is order minimal. Let $I(b)=[c, b]$. Assume $I(b) \supset I(h)=[d, e]$ and $I(b) \neq I(h)$. Since $\overline{\operatorname{Orb}(b)}$ is a minimal set, $c<d$ and $b>e$. This is contradictory to $\phi(a)=b$.

" $\Leftarrow$ ". Let $I(b)=[c, b]$. Then $\phi(c) \leq b$. If $\phi(c)=d<b$, then $I(d) \subset I(b)$ but $I(d) \neq I(b)$. This is contradictory to the order minimality of $b$. 
Let $X \subset \Sigma$ be a subshift, i.e., a closed and invariant subset of $\Sigma$. Denote by

$$
B_{n}(X)=\left\{w \in\{0,1\}^{n}: w \text { occurs in some element of } X\right\} .
$$

Similarly, for any $x \in \Sigma$, we can define

$$
B_{n}(x)=\left\{w \in\{0,1\}^{n}: w \text { occurs in } x\right\} .
$$

Lemma 3.5. Assume that $X$ is a minimal set. If there exists $n>0$ such that $\# B_{n}=\# B_{n+1}$, then $X$ is trivial, i.e., $X$ is a periodic orbit.

Proof. See [1], Theorem 2.11.

Proposition 3.6. Assume that $X$ is a minimal set. If $X$ is nontrivial, then $\sigma$ : $X \rightarrow X$ is not $1-1$.

Proof. Since $X$ is nontrivial, $\# B_{n} \rightarrow \infty$ as $n$ tends to $\infty$. According the above lemma we have $\# B_{n+1}>\# B_{n}$. Hence there exists $w_{n} \in B_{n}$ such that $0 w_{n}, 1 w_{n} \in$ $B_{n+1}$. So let $x_{n}=0 w_{n} u_{n}$ and $y_{n}=1 w_{n} v_{n}$ be in $X$. We may assume that $\lim x_{n}=x$ and $\lim y_{n}=y$. Then $x \neq y$ but $\sigma(x)=\sigma(y)$.

\section{The Image of $\phi$ And Sturmian Sequences}

Lemma 4.1. For any subsets $A, B \subset \Sigma$ we have $\sigma\left(A \cap \sigma^{-1}(B)\right)=\sigma(A) \cap B$.

Proof. Trivial.

Lemma 4.2. For any $x \in \Sigma, I=[0 x, 1 x]$ is an $\mathcal{L}$-interval.

Proof. We only have to show that $\Lambda_{n}=\bigcap_{i>0}^{n} \sigma^{-i} I$ is nonempty. By induction we may assume that $\Lambda_{n} \neq \emptyset$. Now let us show $\bar{\Lambda}_{n+1} \neq \emptyset$.

$$
\begin{aligned}
\Lambda_{n+1} & =\bigcap_{i \geq 0}^{n+1} \sigma^{-i} I \\
& =I \cap \sigma^{-1}\left(\bigcap_{i=0}^{n} \sigma^{-i} I\right) \\
& =I \cap \sigma^{-1} \Lambda_{n} .
\end{aligned}
$$

So, by the above lemma,

$$
\begin{aligned}
\sigma\left(\Lambda_{n+1}\right) & =\sigma(I) \cap \Lambda_{n} \\
& =\sigma\left(\left[0 x, 01^{\infty}\right] \cup\left[10^{\infty}, 1 x\right]\right) \cap \Lambda_{n} \\
& =\left(\left[x, 1^{\infty}\right] \cup\left[0^{\infty}, x\right]\right) \cap \Lambda_{n} \\
& =\Sigma \cap \Lambda_{n}=\Lambda_{n} \\
& \neq \emptyset
\end{aligned}
$$

Corollary 4.3. If $x$ is order minimal, then

(BC) for any $n$ and $B \in\{0,1\}^{n}, 0 B 0$ and $1 B 1$ cannot both occur in $b$.

In particular, only one of 00 and 11 can occur in $b$.

The condition (BC) in the above corollary will be called the Block Condition. Note that according to [1], Lemma 3.06, (BC) is equivalent to the Sturmian block condition there. We have the following simple property for the BC. 
Lemma 4.4. $b \in \Sigma$ satisfies the $B C$ if and only if there exists $z \in \Sigma$ such that $\operatorname{Orb}(b) \subset[0 z, 1 z]$.

Proof. Assume that $\operatorname{Orb}(b) \subset[0 z, 1 z]$ for some $z \in \Sigma$. If both $0 B 0$ and $1 B 1$ occur in $b$ for some $B \in\{0,1\}^{n}$, then there exist $x, y \in \Sigma$ such that $0 B 0 x, 1 B 1 y \in \operatorname{Orb}(b) \subset$ $[0 z, 1 z]$. Hence, $B 0 x \geq z \geq B 1 y$, which is absurd.

Now, assume that $b$ satisfies the BC. Suppose that $I(b)=[0 x, 1 y]$ and $x<y$. Let $i$ be the smallest integer such that $x_{i}=0, y_{i}=1$. Let $B=x_{1} \cdots x_{i-1}=y_{1} \cdots y_{i-1}$. Then both $0 B 0$ and $1 B 1$ occur in $b$, which is contradictory to the $\mathrm{BC}$.

Then let us recall the definition of Sturmian sequence.

For any $\alpha \in(0,1)$, let $J_{\alpha}=[0, \alpha)$ and $\bar{J}_{\alpha}=(0, \alpha]$. For any $x \in[0,1)$ define $x^{\alpha}, \bar{x}^{\alpha} \in \Sigma$ as follows:

$$
\begin{aligned}
x^{\alpha}(i) & = \begin{cases}1, & x+(i-1) \alpha \in J_{\alpha}(\bmod 1), \\
0, & \text { otherwise }\end{cases} \\
\bar{x}^{\alpha}(i) & = \begin{cases}1, & x+(i-1) \alpha \in \bar{J}_{\alpha}(\bmod 1), \\
0, & \text { otherwise }\end{cases}
\end{aligned}
$$

As usual let $M_{\alpha}=\left\{x^{\alpha}, \bar{x}^{\alpha}: x \in[0,1)\right\}$, and then let $M_{0}=\left\{0^{\infty}\right\}$ and $M_{1}=\left\{1^{\infty}\right\}$. For any $\alpha \in[0,1]$, a sequence in $M_{\alpha}$ is called a Sturmian sequence.

Lemma 4.5 ([1]). 1) $M_{\alpha}$ is a minimal set for any $\alpha \in[0,1]$.

2) $x \in \Sigma$ is a Sturmian sequence if and only if $\overline{\operatorname{Orb}(x)}$ is a minimal set and $x$ satisfies the block condition.

So it is clear that

Theorem 4.6. $b \in \Sigma$ is order minimal and $\sigma^{i} b \leq b$ for $i \geq 0$. Then $b$ is a Sturmian sequence. Hence if $b=\phi(a)$, then $b$ is a Sturmian sequence.

Corollary 4.7. $\Lambda$ is a minimal set and contained in $[0 x, 1 x]$ for some $x \in \Sigma$ if and only if $\Lambda$ is a Sturmian minimal set.

\section{Sturmian Sequences and the image of $\phi$ (Continued)}

In the last section we have shown that elements in the image of $\phi$ are all Sturmian sequences. In this section, we show the converse: for every $M_{\alpha}$, the largest element $\bar{\alpha}^{\alpha}$ is in the image of $\phi$. See Theorem 5.5

For any $n \geq 1$ define

$$
\begin{aligned}
& \Lambda_{n}^{0}=\left\{x \in \Sigma: x=10^{n_{1}} 10^{n_{2}} 1 \cdots, n_{i} \in\{n, n+1\}\right\}, \\
& \Lambda_{n}^{1}=\left\{x \in \Sigma: x=1^{n_{1}} 01^{n_{2}} 0 \cdots, n_{i} \in\{n, n+1\}\right\} .
\end{aligned}
$$

And

$$
\Lambda_{n}=\Lambda_{n}^{0} \cup \Lambda_{n}^{1}, \quad \Lambda=\bigcup_{n \geq 1} \Lambda_{n}
$$

And define a natural mapping $f_{n}^{i}: \Lambda_{n}^{i} \rightarrow \Sigma$ as follows:

$$
\begin{aligned}
f_{n}^{0}\left(10^{n_{1}} 10^{n_{2}} 1 \cdots\right) & =\left(n+1-n_{1}\right)\left(n+1-n_{2}\right) \cdots, \\
f_{n}^{1}\left(1^{n_{1}} 01^{n_{2}} 0 \cdots\right) & =\left(n_{1}-n\right)\left(n_{2}-n\right) \cdots .
\end{aligned}
$$

When no confusion will result, we may write $f_{n}^{i}$ as $f_{n}$ or simply $f$. Note that there is one possible confusion for $f$, i.e., $f_{n}\left(1^{n} 01^{n} 0 \cdots\right)=00 \cdots$ but $f_{n-1}\left(1^{n} 01^{n} 0 \cdots\right)=$ $11 \cdots$. 
Remark 5.1. $f_{n}^{i}$ is an order-preserving homeomorphism (1-1, onto and continuous) and maps periodic points to periodic points (but strictly decreases the periods).

Lemma 5.2. If $b$ satisfies $B C, b \neq 0^{\infty}, 10^{\infty}, 1^{\infty}$ and $\sigma^{i} b \leq b$ for $i \geq 0$, then $b \in \Lambda$.

Proof. We may assume that 00 does not occur in $b$. (The other case can be discussed similarly.)

Since $b \neq 1^{\infty}, \sigma^{i} b \leq b$ for all $i \geq 0$ and 00 does not occur in $b, b$ has the form $1^{n_{1}} 01^{n_{2}} 0 \cdots, n_{i}>0$. Obviously, $n_{1} \geq n_{i}$ for all $i>0$. Furthermore, we have $n_{i} \geq n_{1}-1$. In fact, if $n_{i} \leq n_{1}-2$, then $01^{n_{i}} 0$ and $11^{n_{i}} 1 \in B_{n_{i}+2}(b)$, which is impossible since $b$ satisfies the $\mathrm{BC}$.

We need the following lemma (see 3, Theorem 8.1).

Lemma 5.3. $f$ maps Sturmian sequences to Sturmian sequences.

Lemma 5.4. For any $x \in \Sigma$, there exists a unique Sturmian minimal set $M_{\alpha} \subset$ $[0 x, 1 x]$.

Proof. The existence is a consequence of Lemma 3.1, Lemma 4.2 and Theorem 4.6 .

If there are two different Sturmian minimal sets $M_{\alpha}, M_{\beta} \subset[0 x, 1 x]$ with $\alpha \neq \beta$, let $a=\sup M_{\alpha}, b=\sup M_{\beta}$ so that $\sigma^{i} a \leq a$ and $\sigma^{i} b \leq b$ for $i \geq 0$. We may assume that $a<b$ (since $M_{\alpha} \cap M_{\beta}=\emptyset, a \neq b$ ). If $b=1 z$, then $z \leq x$ and so $0 z \leq 0 x$. So, without loss of generality, let $b=1 x$. Since $a<b, \overline{\operatorname{Orb}(a)} \subset[0 x, 1 x)$. So if $1 y \in \overline{\operatorname{Orb}(a)}$, then $1 y<1 x$ and hence $0 y<0 x$ which implies $0 y \notin \overline{\operatorname{Orb}(a)}$ and by Proposition $3.6 \operatorname{Orb}(a)$ is a periodic orbit. Since $a<b, b \neq 1^{\infty}$. We may assume that 00 does not occur in $b$ (another case can be treated similarly). Let $b=1^{n_{1}} 01^{n_{2}} 0 \cdots$. If $n_{1}=1$, then 00 must occur in $a$ and since $a$ is periodic, this will be contradictory to $\sigma^{i}(a) \geq 0 x$. So $n=n_{1}-1>0$. We claim that $a, b \in \Lambda_{n}^{1}$.

In fact, since $\sigma^{j} b \leq b$ for $j \geq 0, n_{i} \leq n+1$. And since $\sigma^{j} b \geq 0 x$ for $j \geq 0, n_{i} \geq n$. So $b \in \Lambda_{n}^{1}$. Since $\sigma^{i} a \geq 0 x, 00$ could not occur in $a$. Since $a$ is periodic, $a$ has the form $1^{m_{1}} 01^{m_{2}} 0 \cdots$. Since $\sigma^{i} a \leq a, m_{i} \leq m_{1}$ for $i \geq 1$. And $a<b$ implies $m_{1} \leq n+1$ and hence $m_{i} \leq n+1$ for $i \geq 1$. And $\sigma^{i} a>0 x$ implies $m_{i} \geq n$ for $i \geq 2$. And hence $m_{1} \geq n$.

Let $f=f_{n}^{1}: \Lambda_{n}^{1} \rightarrow \Sigma$. Since $f$ maps Sturmian sequences to Sturmian sequences and periodic sequences to periodic sequences, $f(a), f(b)$ are Sturmian sequences and $f(a)$ is periodic.

For any $z=1^{s_{1}} 01^{s_{2}} 0 \cdots \in \Lambda_{n}^{1}$, denote by $l_{i}(z)=s_{1}+s_{2}+\cdots+s_{i}+i$ for $i \geq 1$ and $l_{0}(z)=0$.

Let $f(b)=1 y$. Then $f(x)=0 y$. In the following we will show that for $i \geq$ $0, \sigma^{i} f(a), \sigma^{i} f(b) \in[0 y, 1 y]$.

Since $f$ is order-preserving, $\sigma^{i} f(b)=f\left(\sigma^{l_{i}(b)}\right) \leq f(b)$ for $i \geq 0$. Since $\sigma^{l_{i}(b)-1} b \geq$ $0 x, \sigma^{l_{i}(b)} \geq x$, which implies $\sigma^{i} f(b)=f\left(\sigma^{l_{i}(b)} b\right) \geq f(x)=0 y$ for $i>0$. But, obviously, we have $b>x\left(\in \Lambda_{n}^{1}\right)$ and hence $f(b)>f(x)=0 y$.

Similarly, we can show that $\sigma^{i} f(a) \leq 1 y$ for $i \geq 0$ and $\sigma^{i} f(a) \geq 0 y$ for $i>0$. But since $f(a)$ is periodic, we have $f(a) \geq 0 y$.

Since the period of $f(a)$ is strictly less than that of $a$, if we take the pair $(a, b)$ such that the period of $a$ is the smallest, this will be a contradiction.

Theorem 5.5. Let $b \in \Sigma$ be a Sturmian sequence. Then $b$ is order minimal.

Proof. Since $b$ satisfies the Block Condition, $I(b) \subset[0 z, 1 z]$ for some $z \in \Sigma$. Assume that $b$ is not order minimal. Then there is an order minimal $c \in \overline{\mathrm{Orb}(b)}$ such that 
$\sigma^{i} c \leq c$ for $i \geq 0, I(c) \subset I(b)$ and $I(c) \neq I(b)$. So $c$ is a Sturmian sequence according to Theorem 4.6. According to the above lemma, this is impossible.

Corollary 5.6. Assume that $b \in \Sigma$. The following conditions are equivalent.

1) $b=\phi(a)$ for some $a \in \Sigma$.

2) $b$ is order minimal and $\sigma^{i} b \leq b$ for all $i \geq 0$.

3) $b$ is Sturmian sequence and $\sigma^{i} b \leq b$ for all $i \geq 0$.

So now the mapping $\phi$ can be calculated as following.

Theorem 5.7. If $a=1 x \in \Sigma$, then $\phi(a)=1^{\infty}$. If $a=0 x$, then $\phi(a)$ is equal to the Sturmian sequence $b$ such that $\operatorname{Orb}(b) \subset[0 x, 1 x]$ and $\sigma^{i}(b) \leq b$ for all $i \geq 0$.

\section{ACKNOWLEDGEMENT}

The author would like to thank Professor Meirong Zhang, who read the first version of the paper and gave some good suggestions. He also would like to thank the referee for many improvements and the editor for his help.

\section{REFERENCES}

[1] E. M. Coven and G. H. Hedlund. Sequences with minimal block growth. Mathematical Systems Theory 7 (1973), 138-153. MR 48:1199

[2] R. Labarca and S. Plaza, Bifurcation of discontinuous maps of the interval and palindromicnumbers, ICTP preprint IC/98/165, http://www.ictp.trieste.it/ pub_off/

[3] M. Morse and G. A. Hedlund. Symbolic dynamics II: Sturmian trajectories. American Journal of Mathematics 62 (1940), 1-42. MR 1:123d

School of Mathematical Science, Peking University, Beijing 100871, China

E-mail address: gansb@sxx0.math.pku.edu.cn

Current address: The Abdus Salam International Centre for Theoretical Physics, P.O. Box 586, 34100 Trieste, Italy 